\section{Open globe injuries in children: factors predictive of a poor final visual acuity}

A Gupta, I Rahman and B Leatherbarrow

\begin{abstract}
Aims Eye injuries are the leading cause of monocular blindness in children and are challenging to manage. However, limited follow-up studies currently exist. We describe the clinical characteristics and outcomes of open globe injuries presenting to a major UK centre and discuss factors affecting long-term prognosis.
\end{abstract}

Purpose Identification of (1) demographic features, (2) causes, types and location of injuries, (3) initial clinical features and correlation with visual outcome, and (4) predictors of poor visual outcome. Method Retrospective study by reviewing and analysing records of 20 patients, aged 16 years and below, who had undergone repair of an open globe injuries at the Manchester Royal Eye Hospital, UK. Data recorded included demographic data, mechanisms and location of injury, visual acuity, clinical signs on presentation, surgical procedures, postoperative complications, and final visual acuity.

Results The study group comprised of $85 \%$ male subjects, $15 \%$ female subjects. The average age was 9.8 years (range: 1-15). Average follow-up was $\mathbf{1 6 . 3}$ months (range: 3-48 months). Sharp objects (mainly glass or knife) accounted for the majority $(65 \%)$ of injuries. Initial clinical signs associated with poor visual outcomes included poor initial visual acuity, cataract, RAPD, and no initial red reflex. Younger patients and blunt injuries (especially BB gun injuries) had worse visual outcomes.

Conclusion Factors suggesting unfavourable final visual acuity after open globe injuries in children are (1) young age at presentation, (2) poor initial visual acuity, (3) presence of a relative afferent papillary defect, (4) absence of red reflex, (5) cataract, and (6) types and number of surgeries performed.
Awareness and education with regard to prevention of paediatric injuries need to be actively implemented.

Eye (2009) 23, 621-625; doi:10.1038/eye.2008.32; published online 7 March 2008

Keywords: open globe injuries; children; visual outcome

\section{Introduction}

Eye injuries are a leading cause of monocular blindness in children. ${ }^{1-3}$ Children represent a disproportionate percentage of ocular trauma, with estimates of incidence varying between 29 and $35 \% .^{2,3}$ The majority of paediatric ocular trauma cases are due to sporting and recreational activities. ${ }^{1,4}$ Non-penetrating eye injuries account for three quarters of globe injuries in children. ${ }^{1}$ However, open globe injuries may cause profound, irreversible trauma to intraocular structures resulting in an increased risk of visual morbidity. Overall, approximately $43 \%$ of penetrating eye trauma is reported to occur in individuals less than 18 years old. ${ }^{5}$ De Juan et $a l^{5}$ found that children less than 14 years accounted for $36 \%$ of all open globe injuries. Pieramici et al ${ }^{6}$ reported an incidence of $19 \%$ of penetrating eye injuries in children.

The management of open globe injuries in children remains complex in spite of significant advances in surgical techniques. Initial evaluation is difficult due to an often unreliable and variable history, and poor cooperation during ocular examination. Such difficulties often persist during the follow-up period. In addition, postoperative amblyopia remains a difficult management problem.

The aim of our study was to identify the clinical characteristics and outcomes of a series of open globe injuries presenting to a major UK referral centre. We discuss factors affecting long-term prognosis.
Department of Opthalmology, Manchester Royal Eye Hospital, Lister Centre, Manchester, UK

Correspondence: A Gupta, Calderdale

Royal Hospital, Salterheeble, Halifax HX3 OPW

Tel: + 01422357171 ;

Fax: + 4401612765555 .

E-mail: abha@

doctors.org.uk

Received: 7 December 2007 Accepted in revised form: 21 January 2008 Published online: 7 March 2008

This work has been presented as a poster at the Annual Oxford Congress, July 2007 


\section{Methods}

Operating records obtained through a computerised database were used to identify all patients aged 16 years and below, who had undergone repair of an open globe injury from 1 January 1998 to 1 January 2003 at the Manchester Royal Eye Hospital. Case records were reviewed to determine demographic data, including patient age and sex, the mechanisms of ocular injury, and where the injury occurred. The injuries were classified as sharp or blunt. The results of the initial clinical evaluation recorded included best-corrected Snellen visual acuity (BCVA) on presentation, the presence or absence of a relative afferent pupil defect (RAPD), the presence or absence of a red reflex, the presence of anterior segment damage (corneal and/or scleral laceration(s), chemosis, hyphaema, cataract, traumatic mydriasis, an irregular pupil, and iris prolapse), and the presence of posterior segment damage (vitreous haemorrhage, retinal haemorrhage, choroidal rupture or haemorrhage, retinal detachment, and presence of an intraocular foreign body). Surgical procedures, intraoperative and postoperative complications, length of follow-up, and final BCVA were also recorded. Results were statistically analysed using the Fisher's exact test from the statistical program StatsDirect (version 2.6.2, UK).

\section{Results}

A total of 20 cases of open globe injuries in children 16 years or below were identified. All 20 hospital case records were available for review (see Table 1 for a summary of the data). The study group comprised of 17 male subjects and 3 female subjects. The average age of the patients was 9.8 years (range: $1-15$ years). The average follow-up was 16.3 months, median 11.5 months (range: 3-48 months). Of these, one patient was lost to follow-up after 7 months.

Sharp objects caused the majority of the ocular injuries accounting for $13(65 \%)$ cases. These were mainly knife (three cases) or glass (three cases) injuries. The remaining cases included dart (one case), slate (one case), pen/ pencil (two cases) or stick/metal bar (two cases) injuries. Six $(30 \%)$ of the injuries were due to blunt trauma. Three were a result of ball-bearing (BB) gun injuries, one due to the blunt end of a pencil, one secondary to a metal toy and the final case was due to collision with a road sign. In one case, the cause of injury was unknown.

Eyes with a good preoperative VA tended to have a better final VA, although just outside statistical significance $(P=0.09)$. From our results, five $(25 \%)$ cases had a final BCVA of $6 / 60$ or worse. In all of these cases, presenting BCVA was also 6/60 or worse. In five cases, the initial VA was unknown.

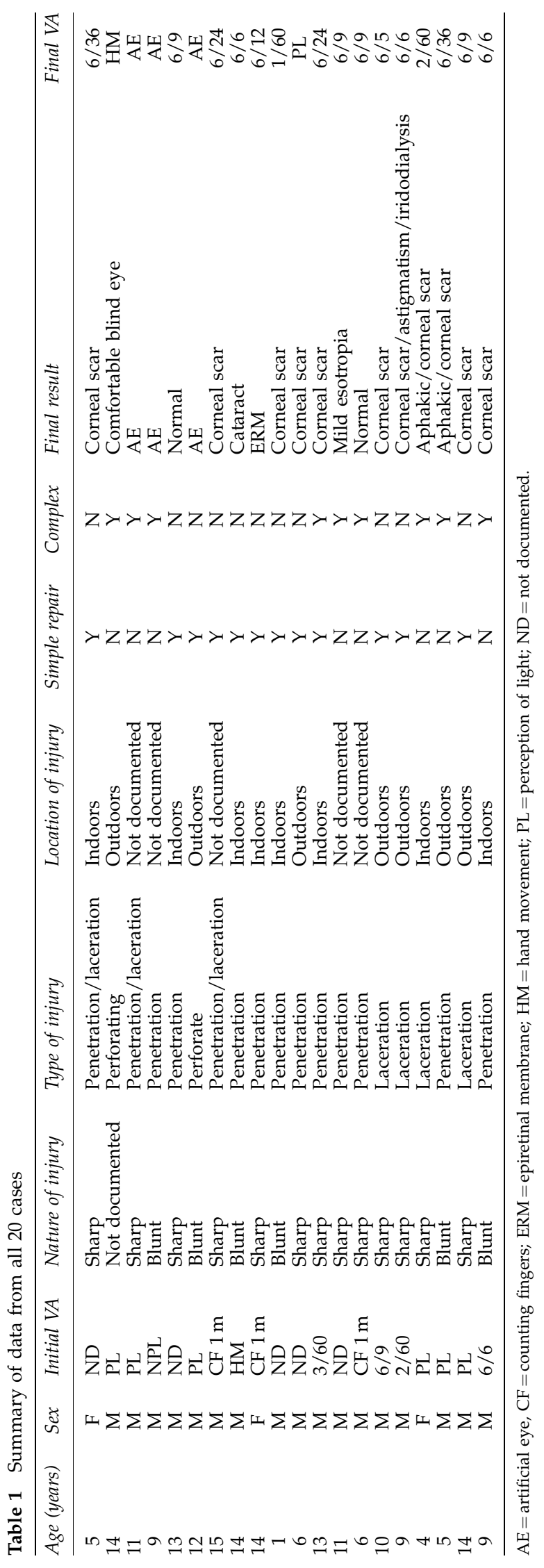


Table 2 Summary of the initial physical findings

\begin{tabular}{lr}
\hline Initial findings & Number \\
\hline Anterior segment & \\
Chemosis & 6 \\
Hyphaema (partial) & 7 \\
Hyphaema (total) & 3 \\
Iris prolapse & 15 \\
Lens abnormalities & 9 \\
Fracture & 1 \\
Corneal/scleral laceration & 19 \\
& \\
Posterior segment & \\
Vitreous haemorrhage & 4 \\
Retinal detachment & 2 \\
Choroidal haemorrhage/rupture & 4 \\
Intraocular foreign body & 2 \\
RAPD & 5 \\
\hline
\end{tabular}

$\mathrm{RAPD}=$ relative afferent pupil defect.

Anterior segment pathology was frequently present. These features are summarised in Table 2. A total of 19 cases had corneal/scleral laceration. Six patients had chemosis on initial presentation and 10 patients suffered a hyphaema, 7 partial and 3 total. Iris prolapse was present in 15 cases. Lens abnormalities were present in nine cases. Four underwent lens removal, of which two remained aphakic and the remaining two had IOL insertion. All cases with a cataract present at initial presentation resulted in a final VA of worse than $6 / 60$ $(P=0.03)$ (Table 2).

Eight children had no red reflex present at the time of initial examination. The absence of a red reflex at initial examination correlated significantly with a poor final VA $(P<0.01)$. Two cases had retinal detachment, four cases had vitreous haemorrhage and a further four cases had choroidal haemorrhage/rupture. Two cases are presented with intraocular foreign bodies.

The presence of a relative afferent pupillary defect was noted in five cases, although not documented in nine cases. The presence of a relative afferent papillary defect was associated with a poorer visual outcome, although marginally outside statistical significance $(P=0.07)$. Of the five cases with an RAPD present at initial examination, four cases achieved a final BCVA 6/60 or worse. Of the six cases that did not have an RAPD presentation, only one case resulted in a vision of $6 / 60$ or worse. Five cases achieved a BCVA better than 6/60 and two cases achieving VA at $6 / 12$ or better. The resulting VA is shown in the Table 3.

Younger patients appeared to have worse visual outcomes from our results $(P=0.06)$. All four children aged 5 years or below had a visual outcome of worse than $6 / 12$, whereas nine children over 5 years achieved a vision of $6 / 12$ or better.
Table 3 Factors predictive of poor final visual acuity

\begin{tabular}{|c|c|c|c|c|}
\hline & $\begin{array}{c}6 / 12 \text { or } \\
\text { better }\end{array}$ & $6 / 15-6 / 60$ & $\begin{array}{c}\text { Worse } \\
\text { than } 6 / 60\end{array}$ & \\
\hline \multicolumn{5}{|l|}{ Initial BCVA } \\
\hline $6 / 12$ or better & 2 & 0 & 0 & $P=0.09$ \\
\hline $6 / 15-6 / 36$ & 0 & 0 & 0 & \\
\hline $6 / 60$ or worse & 5 & 3 & 5 & \\
\hline \multicolumn{5}{|l|}{$R A P D$} \\
\hline Yes & 1 & 0 & 4 & $P=0.07$ \\
\hline No & 2 & 3 & 1 & \\
\hline Unknown & 9 & & & \\
\hline \multicolumn{5}{|l|}{ Age(years) } \\
\hline 5 or less & 0 & 2 & 2 & $P=0.06$ \\
\hline 6 or above & 9 & 2 & 5 & \\
\hline \multicolumn{5}{|c|}{ Mechanism of injury } \\
\hline Blunt & 2 & 1 & 3 & $P=0.29$ \\
\hline Sharp & 7 & 3 & 3 & \\
\hline \multicolumn{5}{|c|}{ Cataract at presentation } \\
\hline Yes & 0 & 0 & 3 & $P=0.03$ \\
\hline No & 9 & 2 & 5 & \\
\hline \multicolumn{5}{|l|}{ Red reflex } \\
\hline Yes & 8 & 2 & 1 & \\
\hline No & 1 & 2 & 5 & $P<0.01$ \\
\hline \multicolumn{5}{|c|}{ Number of operations performed } \\
\hline 1 & 2 & 2 & 0 & \\
\hline 2 & 7 & 3 & 4 & \\
\hline 3 & 0 & 1 & 2 & $P=0.03$ \\
\hline
\end{tabular}

$\mathrm{BCVA}=$ best-corrected Snellen visual acuity; $\mathrm{RAPD}=$ relative afferent pupil defect.

From the six cases resulting in a final VA of worse than $6 / 60$, three cases had injuries caused by sharp objects and three cases had blunt injuries. However, 10 cases (77\%) from the group with sharp injuries resulted in a final VA better than $6 / 36,7(54 \%)$ of which have a final VA of $6 / 12$ or better. This compares to only three cases (50\%) from the blunt injury group achieving a final VA of $6 / 36$ or better, two $(33.3 \%)$ of which achieved a VA of $6 / 12$ or better. This result was not statistically significant $(P=0.29)$.

Surgical repair was simple in 12 cases, 9 cases required complex repair. Simple repair was defined as one necessitating corneal and/or scleral suturing \pm anterior segment reformation. Complex repair was defined as that which warranted a more invasive repair of the globe, for example, lensectomy, vitrectomy, and retinal detachment repair. Patients who had simple surgical repair had better visual outcomes than those with complex repairs. This may reflect the severity of the injury or it may also be due to the extensive scarring and inflammation occurring postoperatively from more numerous procedures. Six cases with simple repair 
resulted in VA of $6 / 12$ or better, two cases had VA between $6 / 15$ and $6 / 60$, and three cases had BCVA worse than $6 / 60$. Three cases with complex repair resulted in VA of $6 / 12$ or better. Four resulted in poor final VA of $6 / 60$ or worse, two of which required subsequent enucleation.

In total, three cases in our series underwent an enucleation. Two of these cases were due to BB gun injuries and one case was due to a sharp injury involving a piece of slate. Two cases had presenting VAs of perception of light and one no perception of light.

We found two cases of endophthalmitis from our results. Both cases had penetrating eye injury with sharp objects. The initial VA was poor (PL) in one case and was not recorded in the notes in the other case. Although treatment was aggressive, the vision did not improve. One case required an enucleation and the other had a final VA of only perception of light. There were no cases of sympathetic ophthalmitis in this series.

\section{Discussion}

Open globe injuries in children are often difficult to assess at the time of presentation and may lead to a poor visual outcome despite best efforts to treat quickly and aggressively.

A higher incidence of trauma in boys than girls is well recognised, ranging from $2.5: 1-5: 1 .^{4,7,8}$ We have shown a male predominance of 5.7:1. Our average age was 9.8 years, comparable to other similar case series.,7 Rostomian et $a l^{8}$, however, reported a much younger average age of 5 years.

Sharp objects accounted for the majority of injuries in our study (65\%). The sharp objects included objects, which are commonly found around the home or school, for example, knife, pen, glass, stick, slate, or dart. As children tend to spend the majority of their time in these locations, this factor may account for the predominance of sharp objects leading to open globe injuries in children. Although not statistically significant, there was a trend towards those with sharp injuries achieving a better final VA compared to those with blunt injuries.

Interestingly, Rahman et $a l^{9}$ found sharp injuries to predominate and result in a better final visual outcome in adults. Others have recognised sharp injuries occurring more commonly in children and hold the view that those injuries have a better visual outcome compared to blunt injuries. ${ }^{4,7,8}$

The influence of amblyogenic factors on final VA is difficult to assess due to the differences in injury type and the small sample size. All children aged 5 years or less had a final BCVA of less than 6/12 compared to $56 \%$ of those 6 years or over who achieved final VAs of better than $6 / 12(P=0.06)$. These results suggest that amblyopia may have a role in the resulting poorer VA in the younger age groups. Rostomian et $a l^{8}$ also noted children's age as being an additional risk factor in poor visual outcome. It is, therefore, extremely important that young children who undergo a repair of an open globe injury are followed up very closely. Aggressive treatment of presumed amblyopia and correction of amblyogenic factors, such as refractive error may improve visual outcome even in children with more severe injuries. It is vital that the child and their families are appropriately counselled and are involved in the often difficult and prolonged treatment of amblyopia. Longer term issues of poor vision need to be addressed to aid in the counselling process, such as future driving and employment restrictions.

It is interesting to note that all the cases with cataract at initial presentation had a poor final BCVA $(P=0.03)$ and would be important to observe if the increasing trend of implantation of lenses in younger age groups improves final VA after open globe injuries in those with resulting lens opacities. Two patients underwent IOL insertion in our series following lens removal.

Initial signs associated with an unfavourable visual outcome include the child's age $(P=0.06)$, initial VA $(P=0.09)$, presence of an RAPD $(P=0.07)$, no initial red reflex $(P<0.01)$, cataract $(P=0.03)$, and the type and number of surgeries performed $(P=0.03)$ (Table 2$)$. These findings correlate well with the literature, ${ }^{5-8,10,11}$ whereby retinal detachment, vitreous haemorrhage, wounds greater than $10 \mathrm{~mm}$ long, and hyphaema have also been associated with poor final VA. ${ }^{7,8,11}$ Only two of our patients had a retinal detachment, the BCVA outcomes of which were 6/12 and HM.

There is a strong relationship between the absence of the red reflex and the final VA, $(P<0.01)$. This finding has not been recorded in previous child-based reviews. The majority of cases without a red reflex also suffered a hyphaema (six out of eight cases). The absence of a red reflex could reflect the severity of the initial injury. This is a relatively straightforward assessment to perform when examining young children and can provide a basis for discussion with the parents with regard to the potential outcome of the eye. We, therefore, suggest that red reflex should be used, along with the other clinical signs mentioned, when examining children with globe injuries to provide an indication for the final visual outcome.

Open globe injuries in children continue to pose a considerable management challenge. In spite of the surgical and clinical developments, the visual potential in damaged eyes is often limited. Initial examination is often difficult as are subsequent follow-up examinations. The severity of the injury, postoperative VA, and visual potential are often difficult to assess. It is hoped that the clinical signs mentioned in this study will aid the 
clinician in examining and following up these children and also provide some clinical indicators towards the final prognosis.

\section{Conclusion}

Penetrating eye trauma remains a frequent and difficult problem. Limited initial assessment of children may prevent adequate prognosis and influence management plans. We have shown that a young age at presentation, poor initial VA, absence of a red reflex, and the presence of cataract significantly influence final outcome. These factors should therefore be assessed to guide treatment planning.

\section{Acknowledgements}

We thank Stephen A Roberts at the University of Manchester for his expert statistical advice concerning this paper.

\section{References}

1 Strahlman E, Elman M, Daub E, Baker S. Causes of paediatric eye injuries. A population-based study. Arch Ophthalmol 1990; 108: 603-606.
2 Maltzman BA, Pruzon H, Mund ML. A survey of ocular trauma. Surv Ophthalmol 1976; 21: 285-290.

3 Niiranen M, Raivio I. Eye injuries in children. $\mathrm{Br} J$ Ophthalmol 1981; 65: 436-438.

4 Jandeck C, Kellner U, Bornfeld N, Foerster MH. Open globe injuries in children. Graefe's Arch Clin Exp Ophthalmol 2000; 238: 420-426.

5 De Juan Jr E, Sternberg Jr P, Michels RG. Penetrating ocular injuries: types of injuries and visual results. Ophthalmology 1983; 90: 1318-1322.

6 Pieramici DJ, MacCumber MW, Humayun MU, Marsh MJ, de Juan Jr E. Open globe injury: update on types of injuries and visual results. Ophthalmology 1996; 103: 1798-1803.

7 Farr AK, Hairston RJ, Humayun MU, Marsh MJ, Pieramici DJ, MacCumber MW et al. Open globe injuries in children: a retrospective analysis. J Paediatr Ophthalmol Strabismus 2001; 38(2): 72-77.

8 Rostomian K, Thach AB, Isfahni A, Pakkar A, Pakkar R, Borchert M. Open globe injuries in children. J APPOS 1998; 2: 234-238.

9 Rahman I, Maino A, Devadason D, Leatherbarrow B. Open globe injuries: factors predictive of poor outcome. Eye 2006; 20(12): 1336-1341.

10 Baxter RJ, Hodgkins PR, Calder I, Morrell AJ, Vardy S, Elkington AR. Visual outcome of childhood anterior perforating eye injuries: prognostic indicators. Eye 1994; 8: 349-352.

11 Rudd JC, Jaeger EA, Freitag SK, Jeffers JB. Traumatically ruptured globes in children. J Paediatr Ophthalmol Strabismus 1994; 31: 307-311. 